\title{
Diagnosing restoration trajectories using demographic modeling and modern coexistence theory
}

\author{
Lina Aoyama ${ }^{1}$, Lauren Shoemaker ${ }^{2}$, Benjamin Gilbert ${ }^{3}$, Sharon Collinge ${ }^{4}$, Akasha Faist $^{5}$, \\ Nancy Shackelford ${ }^{6}$, Vicky Temperton ${ }^{7}$, Gyorgy Barabas $^{8}$, Loralee Larios ${ }^{9}$, Emma \\ Ladouceur $^{10}$, Oscar Godoy ${ }^{11}$, Catherine Bowler ${ }^{12}$, and Lauren Hallett ${ }^{1}$ \\ ${ }^{1}$ University of Oregon \\ ${ }^{2}$ University of Wyoming \\ ${ }^{3}$ University of Toronto \\ ${ }^{4}$ University of Colorado Boulder \\ ${ }^{5}$ New Mexico State University \\ ${ }^{6}$ University of Victoria \\ ${ }^{7}$ Leuphana Universitat Luneburg \\ ${ }^{8}$ Linkopings universitet \\ ${ }^{9}$ University of California Riverside \\ ${ }^{10}$ German Centre for Integrative Biodiversity Research (iDiv) Halle-Jena-Leipzig \\ ${ }^{11}$ Universidad de Cadiz Campus de Puerto Real \\ ${ }^{12}$ The University of Queensland - Saint Lucia Campus
}

July 28, 2021

\begin{abstract}
Restoration success is often measured by comparing target species abundance between restored and reference populations. Abundance may poorly predict long-term success, however, because seed addition may initially inflate restored population abundances, and reference population abundances may fluctuate with environmental variation. A demographic approach, informed by modern coexistence theory, may allow for more accurate diagnosis of restoration trajectories. We modeled population dynamics of an endangered plant (Lasthenia conjugens) in restored vernal pools and compared them to reference populations over 18 years (2000-2017). Model estimates of L. conjugens growth rates were better predictors of long-term trends than observed abundances. Although populations fluctuated in reference pools, annual rainfall variability acted as a stabilizing factor for L. conjugens. In restored pools however, invasive grasses and associated litter accumulation overrode the benefits of environmental variability. Our approach improves assessment of restoration outcomes and indicates when management actions, such as grass removal, will improve future trajectories.
\end{abstract}

\section{Hosted file}

sToration final draft.pdf available at https://authorea.com/users/427939/articles/531999diagnosing-restoration-trajectories-using-demographic-modeling-and-modern-coexistencetheory 